\title{
Quantitatively Controlled Fabrication of Uniaxially Aligned Nanofibrous Scaffold for Cell Adhesion
}

\author{
Suk Hee Park, ${ }^{1}$ Jung Woo Hong,, Jennifer Hyunjong Shin,, ${ }^{1,2}$ and Dong-Yol Yang1 \\ ${ }^{1}$ School of Mechanical Engineering and Aerospace System, Korea Advanced Institute of Science and Technology, \\ Daejeon 305-701, Republic of Korea \\ ${ }^{2}$ Bio and Brain Engineering, Korea Advanced Institute of Science and Technology, Daejeon 305-701, Republic of Korea \\ Correspondence should be addressed to Dong-Yol Yang, dyyang@kaist.ac.kr
}

Received 10 October 2010; Revised 27 January 2011; Accepted 21 March 2011

Academic Editor: Junping Wang

Copyright () 2011 Suk Hee Park et al. This is an open access article distributed under the Creative Commons Attribution License, which permits unrestricted use, distribution, and reproduction in any medium, provided the original work is properly cited.

In light of tissue engineering, development of a functional and controllable scaffold which can promote cell proliferation and differentiation is crucial. In this study, we introduce a controllable collection method of the electrospinning process for regularlydistributed and uniaxially oriented nanofiber scaffold and evaluate the effects of aligned nanofiber density on adhesion of dermal fibroblasts. The suggested spinning collector features an inclined void gap, which allows easy transfer of uniformly aligned fibers onto other surfaces. By undergoing multiple transfers, the density of the nanofibers can be quantitatively controlled. The resultant polycaprolactone (PCL) nanofibers had well-defined nanotopography in a 400-600 nm range. Human dermal fibroblasts were seeded on aligned nanofiber scaffolds of different densities achieved by varying the number of transfers. Cell morphology and actin stress fiber formation was accessed after seven days. The experimental results indicate that the contact guidance of the cells along the fiber alignment can be more activated with more than one guidance feature on a cell; that is, the high density of fiber is attained in so much that fiber spacing gets below the cell size.

\section{Introduction}

The importance of nanostructured topography in view of cell biology has been recognized as one of the key factors in tissue engineering applications. Nano- or micro-architectures that can mimic natural extracellular matrix (ECM) environments have been employed in fabrication of functionalized scaffolds to enhance cellular responses, such as adhesion, migration, proliferation, and locomotion [1-3]. The ECM-like structure, a complex structural network with various fibrillar constituents, has been simulated by a wide variety of fabrication techniques which include MEMS(microelectromechanical system-) based lithography, electrospinning, and imprinting methods [4-6].

The electrospinning process, in particular, has advantages for fabricating cytocompatible scaffolds thanks to its high efficiency and simplicity in producing submicron to nanometer scale fibers from a wide range of biomaterials [7]. When a high electric potential is applied to the pendant drop of a polymer solution, the drop gets deformed into a cone shape, which is known as a Taylor cone. As a charged jet of the polymer solution is launched from the tip of the cone and elongated into thinner fibers, it gradually solidifies forming a nanofibrous mesh on the grounded collector. In typical electrospinning process, the nanofibers from the elongated jet are randomly deposited on the collector due to jet bending and whipping caused by Columbic interaction between the jets [8]. Although these randomly assembled nonwoven nanofiber meshes have a wide range of bioapplications [913], the recent interest in the electrospun nanofibers has been focused on ordered forms of the fibers, especially uniaxially aligned fiber arrays in light of tissue engineering applications for anisotropic tissues such as tendons and muscles. Fiber configuration with uniaxial orientation can be dictated by collector geometry, which has been proposed in various ways including using a rotating cylinder [14, 15], a disk with a sharp edge [16], a spaced wire drum [17], and a parallel electrodes $[18,19]$.

The potential of applying these alignment techniques to scaffold fabrication is significant in that it can provide a defined architecture to guide cellular behavior as well as nanoscaled dimensions for the biomimetic ECM for a wide 
range of cell lines such as fibroblasts, neuron cells, and muscle cells, and so forth [20-27]. Many studies on the guidance effect have been conducted using MEMS-based lithographic techniques to realize precise construction of ridge/groove patterns for cell guidance $[22,23]$. However, in terms of utilization of biocompatible materials for scaffold fabrication and the fabrication itself, the electrospun nanofiber array is far more advantageous than MEMS-based processes. Based on the elctrospinning technique, some efforts have been made to apply the fiber alignment technique with a rotating disk collector [26, 27]. Yet, due to the geometrical limitation of the disk edge, the employed technique makes it difficult to collect a wide area of fiber arrays.

We previously reported on the fabrication process of aligned electrospun nanofibers realized by the proposed inclined gap method [28], which provided regular distribution and high order alignment of the nanofibers over a relatively wide area. The intrinsic instability of fiber landing in the electrospinning process was mitigated by employing two separate strips that were configured horizontally and vertically with the inclined gap. In this study, aligned nanofibrous scaffolds with different densities were prepared by employing multiple transfers of the previously proposed process. Thanks to the favorable feature of void gap with suspended nanofibers, transfers of these fibers onto the other substrate can be easily accomplished. The repetitively overlapping transfer can provide quantitative control of the nanofiber densities in the scaffold in proportion to the number of transfers. Human dermal fibroblasts were cultured for seven days to observe the cell guidance effect and morphological changes on the scaffolds of different densities. Additionally, a smooth PDMS (polydimethylsiloxane) substrate and a number of randomly deposited nanofiber mesh on PDMS were tested to compare with the specimens of aligned nanofibers.

\section{Materials and Methods}

2.1. Materials. Polycaprolactone (PCL, average $M_{n}$ ca. 80,000 ) was obtained by Aldrich (Milwaukee, Wis, USA). Methylene chloride (MC; Junsei Chemical) and N,Ndimethyl formamide (DMF; Junsei Chemical) were used as the solvents for the spinning solutions. PCL was dissolved in a mixture of MC and DMF $(75 / 25 \mathrm{v} / \mathrm{v})$. Cell culturing reagents, fibronectin (FN), Dulbecco's modified Eagle's medium (DMEM), Dulbecco's phosphate buffered saline (DPBS), penicillin-streptomycin, and fetal bovine serum (FBS) were all purchased from Gibco (Carlsbed, CA). Immuno-staining reagents Alexa Fluor 568 phalloidin and DAPI $\left(4^{\prime}, 6\right.$-diamidino-2-phenylindole, dilactate) were obtained from Invitrogen (Carlsbed, Calif, USA).

2.2. Scaffold Fabrication. The schematic of the electrospinning method is illustrated in Figure 1. Polymer solution was prepared by dissolving PCL in the mixture of MC and DMF at the concentration of $20 \mathrm{wt} \%$ defined as a proper condition in the previous work [28]. The higher concentration than that of the typical electrospinning could allow the successful suspension within the inclined void gap due to the sufficiency of the fiber solidification. The PCL solution was electrospun from a 25 -gauge needle connected to a power supply at an applied voltage of $14 \mathrm{kV}$. The infusion rate of solution through the needle was $0.6 \mathrm{~mL} / \mathrm{h}$. The randomly configured nanofiber mesh was obtained by simple electrospinning toward a thin PDMS membrane adhered to slide glass lying on the grounded metal plate. The spinning time was $2 \mathrm{~min}$.

The collector for the formation of uniaxially aligned aluminum strips ( $0.2 \mathrm{~mm}$ thickness), which were fixed horizontally and vertically at upper and lower position, respectively. This height difference between the strips formed the void space and the electrospun nanofibers were suspended along the void gap. The spinning distance from the needle tip to the upper strip was determined as $160 \mathrm{~mm}$. The suspended nanofibers with uniaxial alignment were transferred onto a surface of the PDMS membrane adhered to the slide glass and both ends of the fiber array were anchored with Permount (Fisher Scientific, Pittsburgh, Pa, USA).

2.3. Characterization of Electrospun Nanofibers. The resultant samples that were transferred with electrospun nanofibers were photographed using a scanning electron microscope (SEM; JSM-6300, JEOL) and an optical microscope (iCamscope, Sometech Vision). The fiber alignment was quantified by employing the angles between the desired direction (perpendicular to the edge lines of two strips) and the longitudinal axes of the fibers. The angle distribution of each nanofiber was measured from the captured images by using ImageTool 3.0 (University of Texas Health Science Center in San Antonio, Tex, USA).

2.4. Cell Culture. Cell studies were performed using human dermal fibroblasts (6th and 10th passage) purchased from Lonza (Basal, Switzerland). The cells were cultured in Dulbecco's modified Eagle's medium (DMEM) (Lonza, Basal, Switzerland) containing $10 \%$ fetal bovine serum (FBS) supplemented with penicillin-streptomycin (Sigma, St. Louis, Mo, USA). Cultures were incubated in a humidified atmosphere of $5 \% \mathrm{CO}_{2}$ in air at $37^{\circ} \mathrm{C}$. Prior to the cell seeding, all nanofiber scaffolds were sterilized in ethanol for 24 hours under ultraviolet irradiation, treated with $\mathrm{O}_{2}$ plasma for 2 mins (Femto Science) and coated with fibronectin at $10 \mu \mathrm{g} / \mathrm{mL}$ for 1 hour at room temperature. Cells were harvested using trypsin/EDTA and resuspended in DMEM. Cells were then seeded onto the scaffolds at its density of $10^{4}$ cells per scaffold and incubated for 7 days in appropriate growth conditions. For mature binding of cells to the substrate, first media change was done after 36 hours of cell seeding and an additional media change was done on the 5 th day for optimal growth. The process was carried out with extreme care to minimize any possible undesired simulation or dislodging of cells by mechanical agitation.

2.5. Immunofluorescence. After experiments, cells were washed with the warmed DPBS and fixed for $15 \mathrm{~min}$ in 3.7\% formaldehyde. After DPBS washing, cells were permeabilized for $20 \mathrm{~min}$ in $2 \%$ Triton X-100 and blocked in 3\% BSA (Invitrogen). Following three washes in DPBS, cells were 


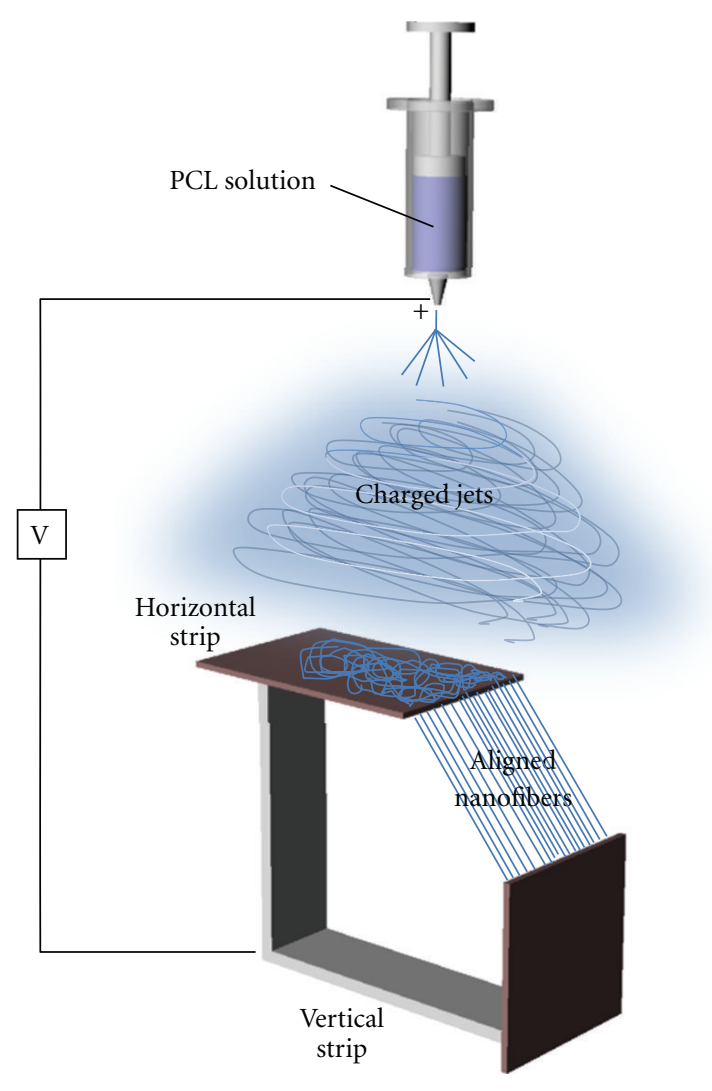

(a)

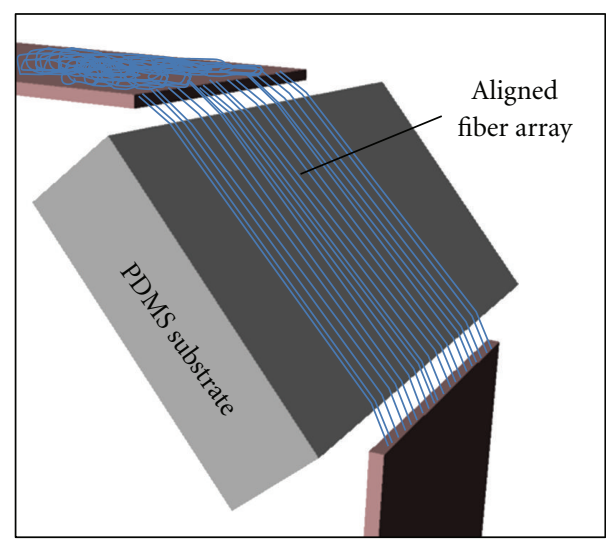

(b)

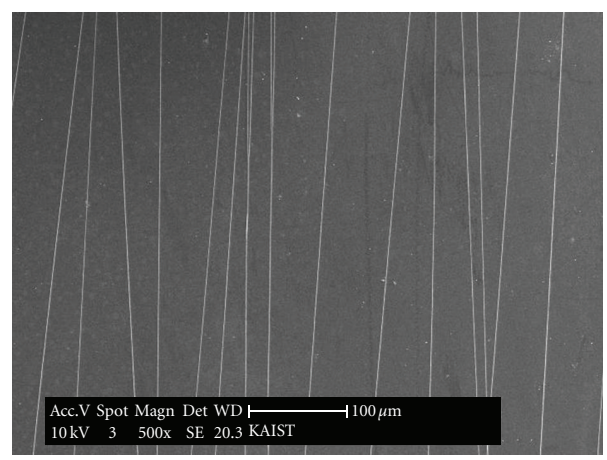

(c)

FIGURE 1: (a) Schematic diagram of the electrospinning setup for the inclined gap method. (b) The transfer of collected fibers onto PDMS substrate. (c) SEM image of the transferred nanofiber array.

stained for F-actin cytoskeleton with Alexa-568-phalloidin (Molecular Probes) at 1:50 dilution in blocking buffer. After rinsing, the nuclei of the cells were labeled with DAPI (300 nM, D1306, Molecular Probes), and then, they were finally mounted in a Vectashield $(\mathrm{H}-1000$, Vector Laboratories) to minimize photobleaching. The cells were imaged using a Zeiss fluorescence microscope (Axiovert200M, Zeiss) equipped with a CCD camera (Axiocam HSM, Zeiss) and image analysis software (Axiovision, Zeiss).

2.6. Cell Morphology. After 7 days of cell culture, the stained cells were observed under a light microscope (Carl Zeiss Axiovert $200 \mathrm{M}$ ) with phase contrast. 60 cells from each scaffold were randomly selected and the fluorescent images of actin and nuclei were analyzed using ImageTool 3.0 to measure the extent of cell alignment. The orientation angle of cell long axis was measured with respect to the reference, which is the desired direction of fiber alignment, that is, perpendicular axis to the collector edges. In addition, cell elongation factor $E_{f}$ was calculated by [29]. The values of the elongation factor were calculated from 30 cells randomly selected from each scaffold. All statically analyses were performed using the Student's $t$-test. Mean values from different independent experiments were calculated and expressed as the mean \pm STD.

\section{Results and Discussion}

3.1. Uniformity of Nanofiber Array. In order to improve the uniformity of the aligned nanofiber array, the fiber collector was modified from a conventional design for the general gap method [28]. There have been several techniques developed to fabricate uniaxially oriented nanofibers [1419]. Especially, the gap method, where two parallel electrodes are used as the collector, is considered to be effective in producing an aligned nanofiber array covering a wide area. When the two separate electrodes are placed at the position of the collector with a gap in between, the electric field lines are split into each edge of the electrodes. Because the electrospun jets tend to travel along the field lines, as-spun fibers are longitudinally stretched toward both edges of the electrodes and become uniaxially suspended across the gap $[18,19]$.

As reported previously [28], the modification of the configuration from a planar gap to an inclined gap allowed flexibility in time and space for improved alignment effect. As shown in Figures 2(a)-2(d) fiber collection from the inclined gap serves to create better conditions in both the alignment of fiber angle and the homogeneity in fiber distribution, whereas irregular distribution of nanofibers was observed in the case of the planar gap (marked by arrows in Figures 2(b), $2(\mathrm{~g})$, and $2(\mathrm{~h})$ ). These irregularities would be accumulative 
as the number of transfers increased, as shown in Figures $2(\mathrm{~g})$, and $2(\mathrm{~h})$. Thus, since multiple transfers were necessary to create a nanofiber array of higher density, the use of inclined gap configuration would be more advantageous in generating more homogenous scaffolds

3.2. Controlled Quantity of Nanofibers. Although the utilized electrospinning technique has the advantage of producing the relatively large area of fiber array, repulsion caused by the residual charges on the collected fibers creates an inherent difficulty in generating controlled nanofibrous scaffold containing a sufficient quantity of fibers. As shown in Figure 1(c), most of the intervals between the fibers appear to be several tens of micrometer which is larger than typical eukaryotic cells; for instance, the size of a fibroblast is about $10 \mu \mathrm{m}$ in length and $2-3 \mu \mathrm{m}$ in width [30]. Thus, a subten-micrometer scale of fiber distance would be desired to ensure the interaction between the cell and the nanofibers. By employing multiple transfers on an inclined configuration, a reduction of fiber distance can be attained at approximately $2 \mu \mathrm{m}$ when 20 transfers were carried out (Figure $3(\mathrm{~d})$ ). As the quantity of nanofibers collected from single-step electrospinning was nearly invariable for the determined spinning time of $30 \mathrm{sec}$, the fiber quantity within the array would be proportional to the number of transfers. These results confirm the feasibility of the quantitative control of nanofibers using multitransfers of nanofibers using the inclined configuration of collector (Figures 2(a), 2(c), 2(e), and 2(f)).

3.3. Morphology of Nanofibers. While conventional electrospinning uses a simple electrically grounded collector without a gap, the collector used in our method comprises of two aluminum sheets separated by a gap. Because this collector configuration with a gap is designed such that asspun fibers become suspended between both edges of the sheets, relatively rigid jets and fibers are preferred for stable suspension. When the initial concentration of the utilized solution is high, the whole jets and fibers throughout the spinning process exhibit more solid-like behavior. Thus, we increased the solution concentration to $20 \mathrm{wt} \%$ from the typical concentration of $10-12 \mathrm{wt} \%$.

One concern about increasing the solution concentration for better mechanical stability is its consequence in the increased diameter of the nanofibers [31]. In our process, however, the fiber diameter can be achieved within the submicrometer scale despite the relatively high solution concentration. While the fiber deposited directly on the upper strip exhibited a thicker diameter (typically of the order of few micrometers as shown in Figure 4(b)), the nanofibers suspended between the edges of the upper and lower strips featured thinner diameters (typically ranging from 400 to $600 \mathrm{~nm}$ as shown in Figure 4(c)) suitable for tissue engineering applications.

3.4. Cell Morphology. In order to test how substratum topographies generated from the transferred nanofiber scaffolds influence cell morphology, several specimens of different densities of nanofibers were prepared by varying number of transfers from 1, 3, to 10 . Fibroblasts were seeded and cultured for seven days on each scaffold. As shown in Figure 5, the degree of cell alignment on the substrate transferred with nanofibers showed positive correlations with the density of fibers. Cells cultured on the scaffolds of 3 and 10 transfers show elongated morphology in the direction parallel to fiber alignment, whereas those on the scaffold of single transfer show little tendency of orientation.

To visualize the interaction between the nanofibers and the cultured cells better, cells were fluorescently stained for nuclei and cytoskeletal actin with DAPI and rhodamine phalloidin, respectively. As a control, a smooth PDMS substrate without nanofibers (Figure 6(a)) and the randomlyelectrospun nanofiber scaffolds (Figure 6(b)) were used and compared with the aligned nanofiber substrates of different densities (Figures 6(c)-6(e)). All substrates were coated with fibronectin under the identified condition as described in Section 2. The superimposed stack of phase contrast and fluorescent images for nuclei and actin show intimate association of cells with nanofibers (Figures 6(b)-6(e)). The case of randomly oriented fibers shown in Figure 6(b) indicates cells' preference for the fibers to the smooth underlying surface. Often, cells stretch along the fiber length and are bounded by neighboring fibers. However, due to its randomness in orientation, neither of the cell alignment distribution nor the elongation morphology was any different from those cultured on the smooth PDMS surface without fibers.

On the scaffold with a single transfer (Figure 6(c)), the cell alignment $\left(55 \%\right.$ cells within $\pm 15^{\circ}$ of the reference direction) and elongation (the average value of 5.8) were achieved to some extent, but a significant fraction of cells was shown to be unaffected by nanofibers due to large fiberfiber distance. On the other hand, cells on the scaffolds with 5 and 20 transfers, as shown in Figures 6(d) and 6(e), showed clear alignment and elongation along the fibers. For the scaffolds with 5 transfers, $67 \%$ of cells aligned within $\pm 15^{\circ}$ of the reference direction and the average elongation factor was 6.8 , whereas the scaffolds with 20 transfers showed $95 \%$ alignment and the elongation factor of 11.6. Most of the aligned cells span over more than two fibers across its body width. It is noteworthy that the cell guidance appeared to be more effective when the fiber spacing in the scaffold was similar to or smaller than the cell size. In other words, cell contact with the multiple guide topographies may be crucial for contact guidance effect.

Figure 7 shows the formations of actin stress fibers of the cells cultured on the scaffolds with different number of nanofiber transfers. Cells making multiple contacts with the scaffold show strongly visible and densely distributed stress fibers as shown in Figures 7(c), and 7(d), compared to those forming a single contact with the scaffold shown in Figures $7(\mathrm{a})$, and $7(\mathrm{~b})$. Because the actin stress fiber formation is one of the critical components in cellular functions such as migration and differentiation, the fiber density control would be an important parameter for the scaffold design for tissue engineering.

This study presents an initial effort to apply uniaxially aligned and uniformly distributed nanofibers to scaffoldbased tissue engineering in a quantitatively controlled 


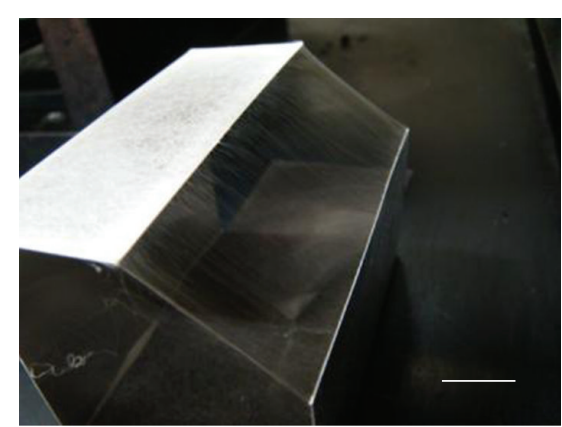

(a)

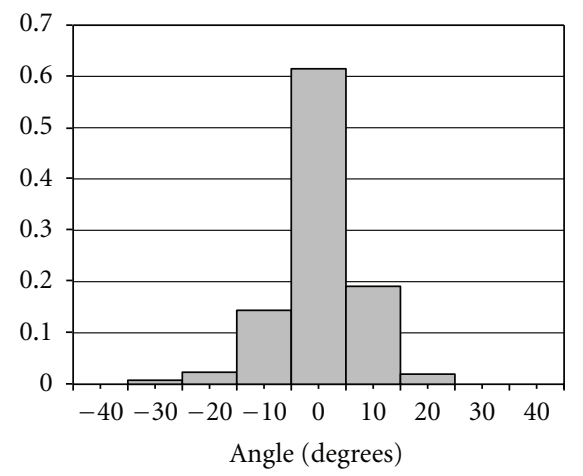

(c)

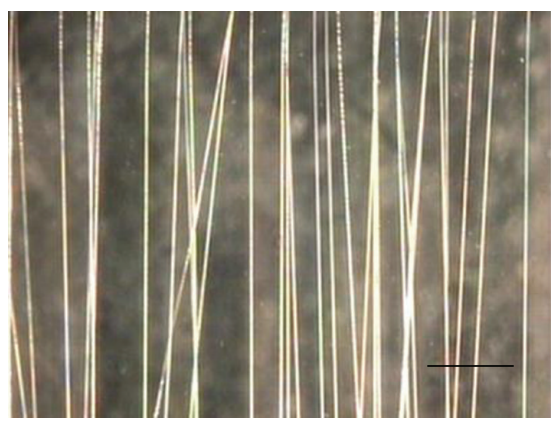

(e)

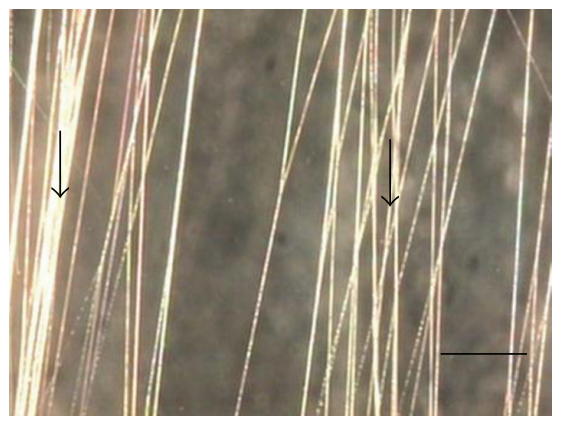

(g)

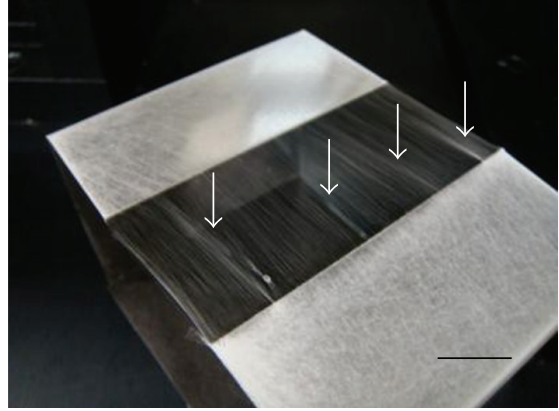

(b)

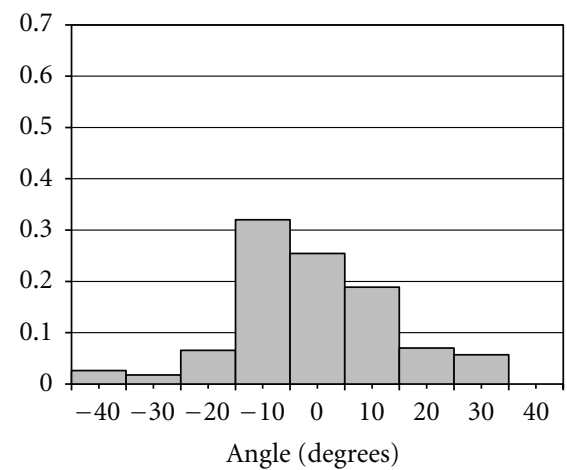

(d)

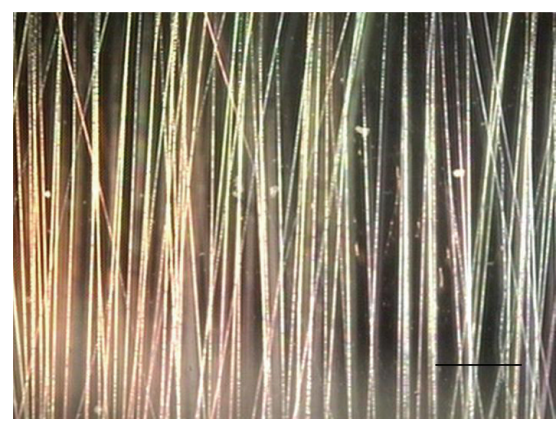

(f)

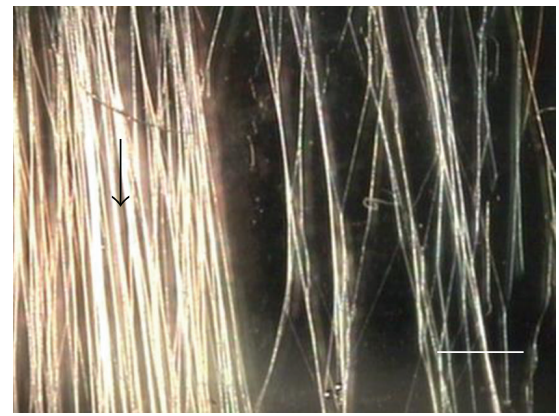

(h)

FIGURE 2: Comparison between results of the inclined and the planar gap collection: photographs of the fiber arrays suspended on (a) the inclined gap collector and (b) the planar gap collector. Histograms of angle distribution of fiber alignment in the arrays transferred from (c) the inclined gap collector and (d) the planar gap collector. Homogeneous fiber arrays collected from the inclined gap by (e) single transfer and (f) 3 transfers. Inhomogeneous fiber arrays collected from the planar gap by (g) single transfer and (h) 3 transfers. Arrows indicate the inhomogeneous conditions of the fiber distributions. The scale bars are $10 \mathrm{~mm}(\mathrm{a}, \mathrm{b})$ and $100 \mu \mathrm{m}(\mathrm{e})-(\mathrm{h})$, respectively. 


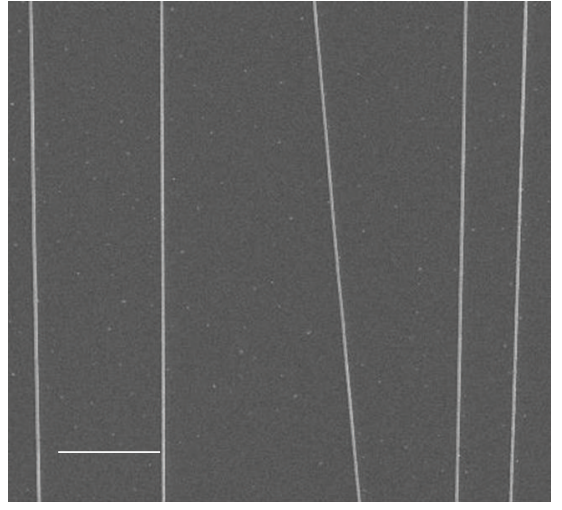

(a)

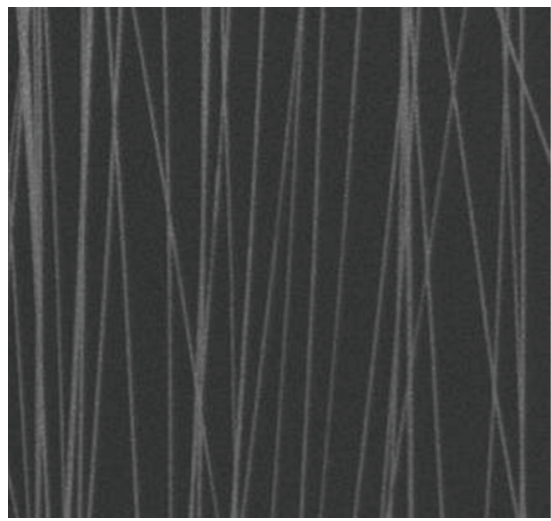

(c)

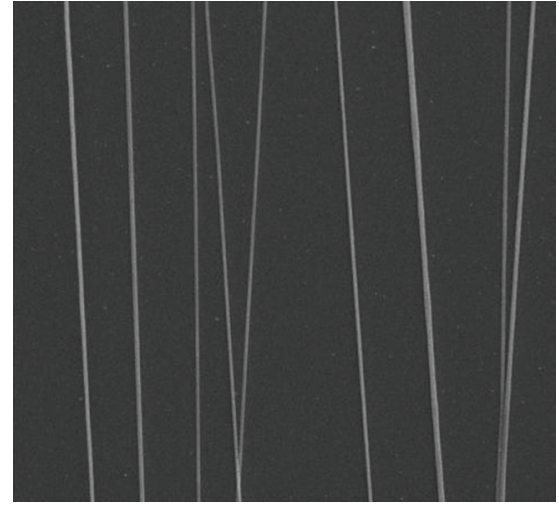

(b)

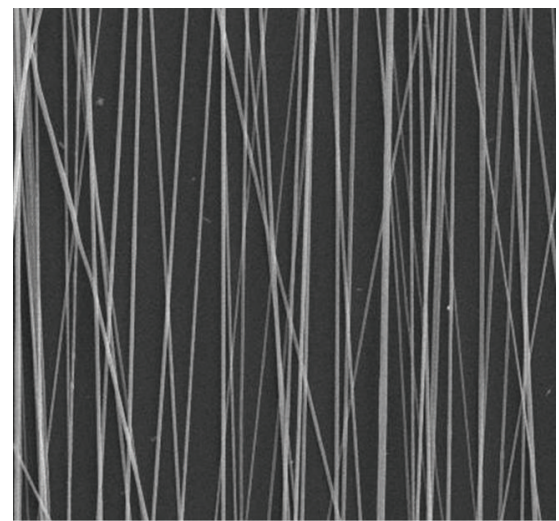

(d)

FIGURE 3: SEM images of the nanofiber arrays transferred repetitively: (a) 1 transfer, (b) 3 transfers, (c) 10 transfers, and (d) 20 transfers. The scale bar on (a) is $20 \mu \mathrm{m}$, and the other images are at the same magnification.

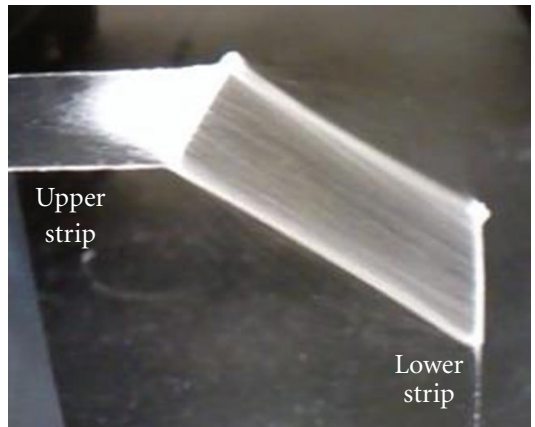

(a)

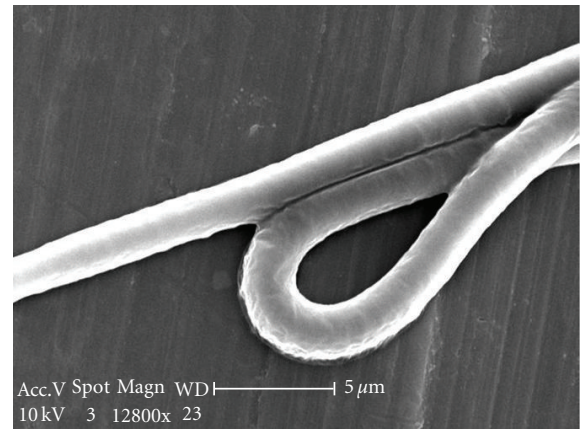

(b)

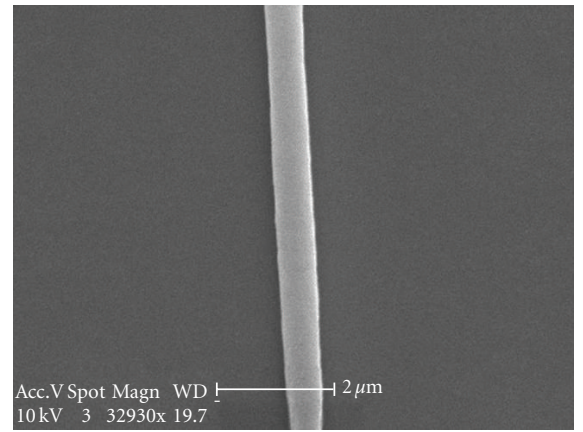

(c)

FIGURE 4: (a) Photograph of nanofibers collected on the upper strip and within the gap space. SEM images of (b) the microscale fibers deposited directly on the upper strip (the scale bar is $5 \mu \mathrm{m}$ ), and (c) the submicroscale fiber uniaxially suspended within the gap space (the scale bar is $2 \mu \mathrm{m}$ ).

manner and implies that a highly dense scaffold with nanofiber alignment would have functional significance. Besides as a dermal fibroblast used for wound healing strategy, the developed nanofiber scaffold could potentially contribute to other tissue engineering applications where tissue anisotropy is a critical factor (e.g., blood vessels, muscles and nerves), and thus other cell types should be tested for efficacy of the scaffolds. In addition, because of the material universality of the electrospinning process, naturally occurring polymers such as collagens can be tested as scaffold materials. 


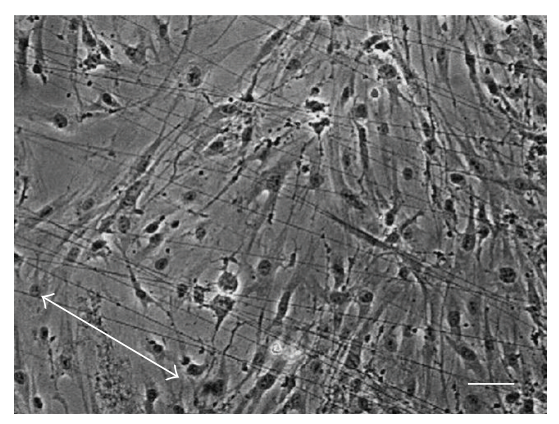

(a)

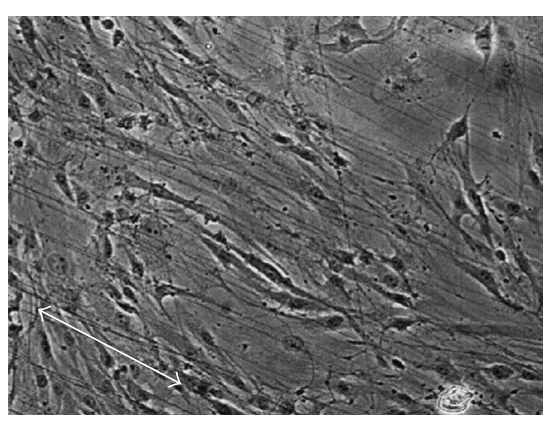

(b)

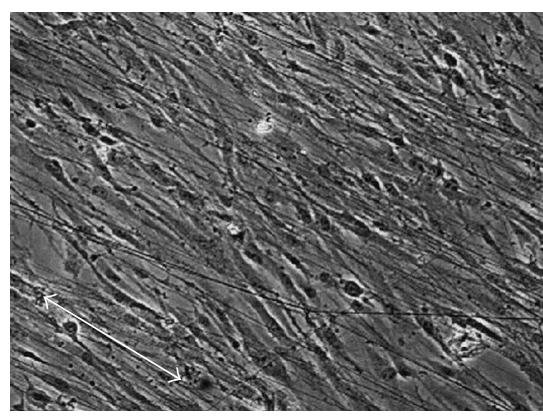

(c)

Figure 5: Phase contrast micrographs illustrating the cell alignment effect of different fiber arrays for (a) 1, (b) 3, and (c) 10 transfers. The scale bar on (a) is $100 \mu \mathrm{m}$; the other images are at the same magnification. The arrows indicate the direction of the fiber orientation.
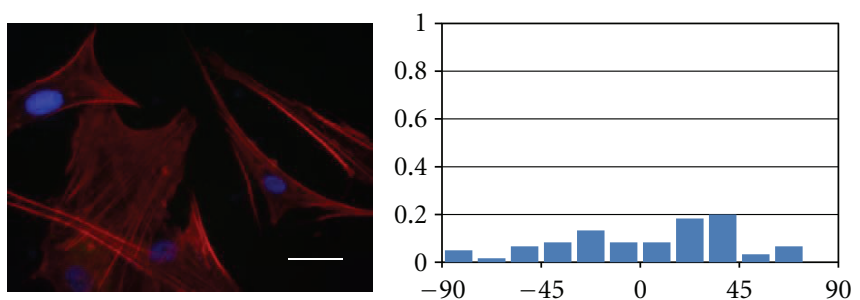

(a)
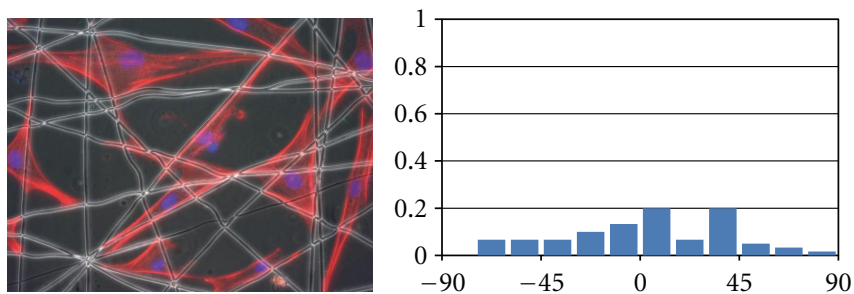

(b)
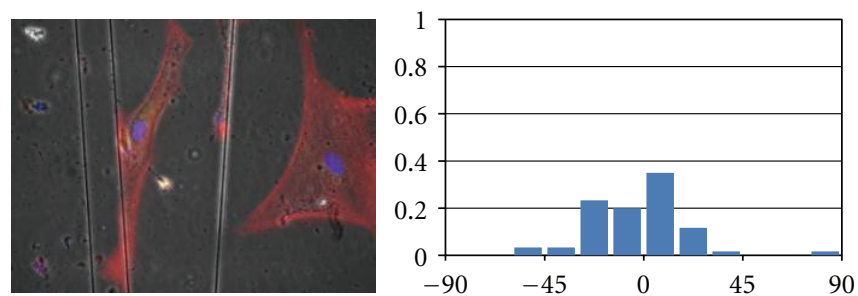

(c)
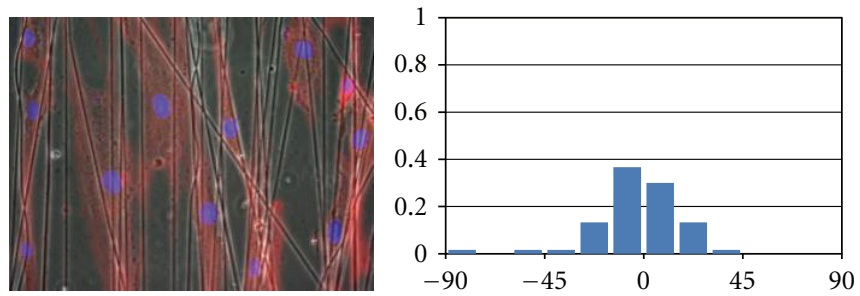

(d)
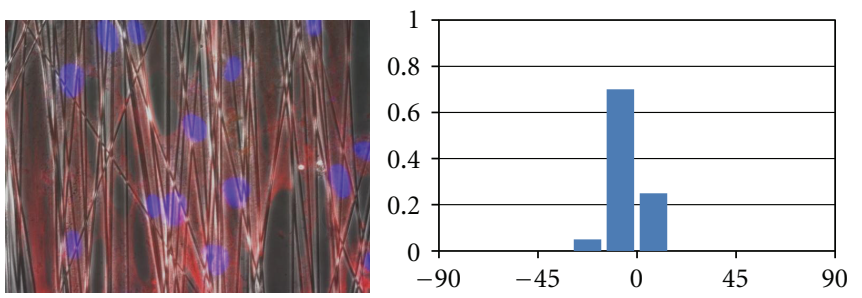

(e)

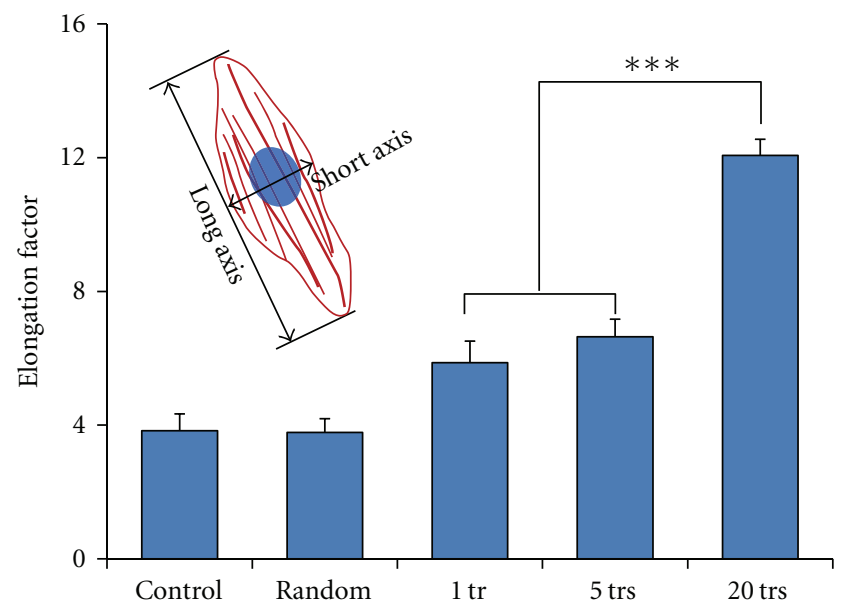

$($ Elongation factor $)=($ long axis $) /($ short axis $)-1$

(f)

FIGURE 6: Immunofluorescent staining images of cell morphologies and quantitative assessments of cell alignments on (a) smooth PDMS substrate without fiber (control), (b) fiber mesh deposited randomly (random), (c) singly transferred substrate with aligned nanofibers ( $1 \mathrm{tr}$ ), and multiply transferred substrate with (d) 5 ( $5 \mathrm{trs}$ ) and (e) 20 transfers ( $20 \mathrm{trs}$ ). Red and blue correspond to actin and nucleus, respectively. The scale bar on (a) is $50 \mu \mathrm{m}$; the other images are at the same magnification. (f) Elongation factor $\left(E_{f}\right)$ of the each scaffold. Data shown as mean \pm standard error $\left(n=30\right.$ in each scaffold, $\left.{ }^{* * *} P<.001\right)$. 


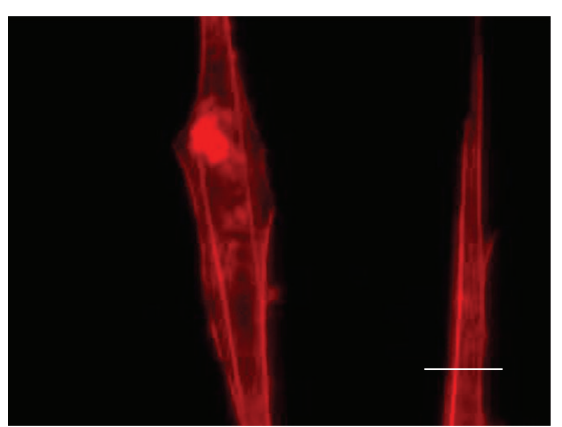

(a)

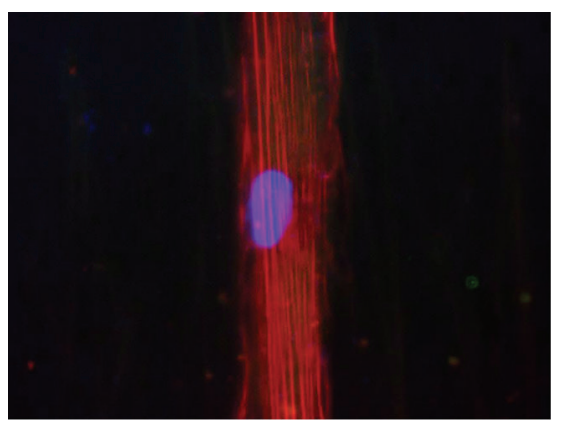

(c)

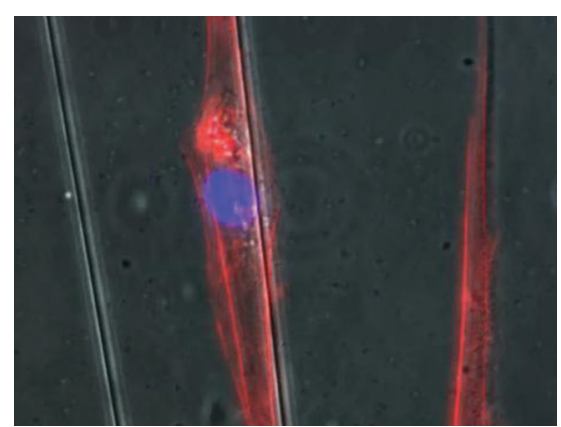

(b)

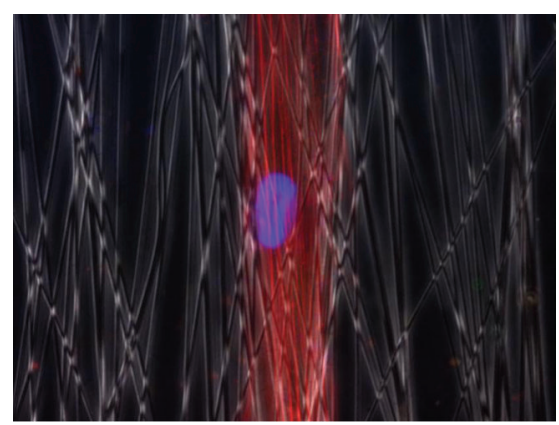

(d)

FIGURE 7: Immunofluorescent staining images to visualize the formation of actin stress fiber on the substrate (a, b) with single transfer and (c, d) with 20 transfers. The scale bar on (a) is $30 \mu \mathrm{m}$; the other images are at the same magnification.

\section{Conclusions}

In this study, uniaxially electrospun nanofiber arrays were constructed with quantitatively controlled density. As a single transfer of the nanofibers collected from the inclined gap provided a well-aligned and regularly distributed form, the developed nanofiber array could be applied to tissue scaffold by employing multiple transfers. The fiber density within a scaffold could be controlled in proportion to the number of transfers. Diameters of the nanofibers in the scaffold were in a submicrometer range, which means that the fibers were appropriate to utilize as nanotopographic features. In regard to the contact guidance along the aligned direction of the nanofibers, favorable interactions between the fibroblasts and the developed scaffold, especially in a highly dense scaffold with the fiber spacing reduced down to less than cell size, were revealed in observation of cell morphologies. The results regarding the aspect of the fiber density control are believed to help practical scaffold design with aligned configuration. Further studies with other cell types utilizing more quantitative biochemical assays are under way to evaluate the feasibility of the developed scaffolds to tissue engineering applications.

\section{References}

[1] C. S. Chen, M. Mrksich, S. Huang, G. M. Whitesides, and D. E. Ingber, "Geometric control of cell life and death," Science, vol. 276, no. 5317, pp. 1425-1428, 1997.

[2] R. G. Flemming, C. J. Murphy, G. A. Abrams, S. L. Goodman, and P. F. Nealey, "Effects of synthetic micro- and nanostructured surfaces on cell behavior," Biomaterials, vol. 20, no. 6, pp. 573-588, 1999.

[3] J. Y. Lim and H. J. Donahue, "Cell sensing and response to micro- and nanostructured surfaces produced by chemical and topographic patterning," Tissue Engineering, vol. 13, no. 8, pp. 1879-1891, 2007.

[4] Y. Lu and S. C. Chen, "Micro and nano-fabrication of biodegradable polymers for drug delivery," Advanced Drug Delivery Reviews, vol. 56, no. 11, pp. 1621-1633, 2004.

[5] A. Khademhosseini, R. Langer, J. Borenstein, and J. P. Vacanti, "Microscale technologies for tissue engineering and biology," Proceedings of the National Academy of Sciences of the United States of America, vol. 103, no. 8, pp. 2480-2487, 2006. 
[6] V. Mironov, V. Kasyanov, and R. R. Markwald, "Nanotechnology in vascular tissue engineering: from nanoscaffolding towards rapid vessel biofabrication," Trends in Biotechnology, vol. 26, no. 6, pp. 338-344, 2008.

[7] Z. M. Huang, Y. Z. Zhang, M. Kotaki, and S. Ramakrishna, "A review on polymer nanofibers by electrospinning and their applications in nanocomposites," Composites Science and Technology, vol. 63, no. 15, pp. 2223-2253, 2003.

[8] A. L. Yarin, S. Koombhongse, and D. H. Reneker, "Bending instability in electrospinning of nanofibers," Journal of Applied Physics, vol. 89, no. 5, pp. 3018-3026, 2001.

[9] W. J. Li, C. T. Laurencin, E. J. Caterson, R. S. Tuan, and F. K. Ko, "Electrospun nanofibrous structure: a novel scaffold for tissue engineering," Journal of Biomedical Materials Research, vol. 60, no. 4, pp. 613-621, 2002.

[10] C. Xu, R. Inai, M. Kotaki, and S. Ramakrishna, "Electrospun nanofiber fabrication as synthetic extracellular matrix and its potential for vascular tissue engineering," Tissue Engineering, vol. 10, no. 7-8, pp. 1160-1168, 2004.

[11] L. S. Nair, S. Bhattacharyya, and C. T. Laurencin, "Development of novel tissue engineering scaffolds via electrospinning," Expert Opinion on Biological Therapy, vol. 4, no. 5, pp. 659-668, 2004.

[12] S. Y. Chew, Y. Wen, Y. Dzenis, and K. W. Leong, "The role of electrospinning in the emerging field of nanomedicine," Current Pharmaceutical Design, vol. 12, no. 36, pp. 4751-4770, 2006.

[13] S. G. Kumbar, R. James, S. P. Nukavarapu, and C. T. Laurencin, "Electrospun nanofiber scaffolds: engineering soft tissues," Biomedical Materials, vol. 3, no. 3, Article ID 034002, 15 pages, 2008.

[14] E. D. Boland, G. E. Wnek, D. G. Simpson, K. J. Pawlowski, and G. L. Bowlin, "Tailoring tissue engineering scaffolds using electrostatic processing techniques: a study of poly(glycolic acid) electrospinning," Journal of Macromolecular Science, vol. 38, no. 12, pp. 1231-1243, 2001.

[15] J. A. Matthews, G. E. Wnek, D. G. Simpson, and G. L. Bowlin, "Electrospinning of collagen nanofibers," Biomacromolecules, vol. 3, no. 2, pp. 232-238, 2002.

[16] A. Theron, E. Zussman, and A. L. Yarin, "Electrostatic fieldassisted alignment of electrospun nanofibres," Nanotechnology, vol. 12, no. 3, pp. 384-390, 2001.

[17] P. Katta, M. Alessandro, R. D. Ramsier, and G. G. Chase, "Continuous electrospinning of aligned polymer nanofibers onto a wire drum collector," Nano Letters, vol. 4, no. 11, pp. 2215-2218, 2004.

[18] D. Li, Y. Wang, and Y. Xia, "Electrospinning of polymeric and ceramic nanofibers as uniaxially aligned arrays," Nano Letters, vol. 3, no. 8, pp. 1167-1171, 2003.

[19] D. Li, Y. Wang, and Y. Xia, "Electrospinning Nanofibers as Uniaxially Aligned Arrays and Layer-by-Layer Stacked Films," Advanced Materials, vol. 16, no. 4, pp. 361-366, 2004.

[20] P. Weiss, "In vitro experiments on the factors determining the course of the outgrowing nerve fiber," Journal of Experimental Zoology, vol. 68, no. 3, pp. 393-448, 1934.

[21] J. Sutherland, M. Denyer, and S. Britland, "Contact guidance in human dermal fibroblasts is modulated by population pressure," Journal of Anatomy, vol. 206, no. 6, pp. 581-587, 2005.

[22] C. A. Mills, J. Escarré, E. Engel et al., "Micro- and nanostructuring of poly(ethylene-2,6-naphthalate) surfaces, for biomedical applications, using polymer replication techniques," Nanotechnology, vol. 16, no. 4, pp. 369-375, 2005.
[23] A. I. Teixeira, G. A. Abrams, P. J. Bertics, C. J. Murphy, and P. F. Nealey, "Epithelial contact guidance on well-defined microand nanostructured substrates," Journal of Cell Science, vol. 116, no. 10, pp. 1881-1892, 2003.

[24] G. A. Dunn and A. F. Brown, "Alignment of fibroblasts on grooved surfaces described by a simple geometric transformation," Journal of Cell Science, vol. 83, pp. 313-340, 1986.

[25] P. Clark, P. Connolly, and G. R. Moores, "Cell guidance by micropatterned adhesiveness in vitro," Journal of Cell Science, vol. 103, no. 1, pp. 287-292, 1992.

[26] C. Y. Xu, R. Inai, M. Kotaki, and S. Ramakrishna, "Aligned biodegradable nanofibrous structure: a potential scaffold for blood vessel engineering," Biomaterials, vol. 25, no. 5, pp. 877886, 2004.

[27] J. M. Corey, C. C. Gertz, B. S. Wang et al., "The design of electrospun PLLA nanofiber scaffolds compatible with serumfree growth of primary motor and sensory neurons," Acta Biomaterialia, vol. 4, no. 4, pp. 863-875, 2008.

[28] S. H. Park and D. Y. Yang, "Fabrication of aligned electrospun nanofibers by inclined gap method," Journal of Applied Polymer Science, vol. 120, no. 3, pp. 1800-1807, 2011.

[29] A. S. Crouch, D. Miller, K. J. Luebke, and W. Hu, "Correlation of anisotropic cell behaviors with topographic aspect ratio," Biomaterials, vol. 30, no. 8, pp. 1560-1567, 2009.

[30] G. Khang, S. J. Lee, J. H. Lee, and H. B. Lee, "Interaction of Fibroblast Cells onto Fibers with Different Diameter," Korea Polymer Journal, vol. 7, no. 2, pp. 102-107, 1999.

[31] C. A. Bashur, L. A. Dahlgren, and A. S. Goldstein, "Effect of fiber diameter and orientation on fibroblast morphology and proliferation on electrospun poly(d,1-lactic-co-glycolic acid) meshes," Biomaterials, vol. 27, no. 33, pp. 5681-5688, 2006. 

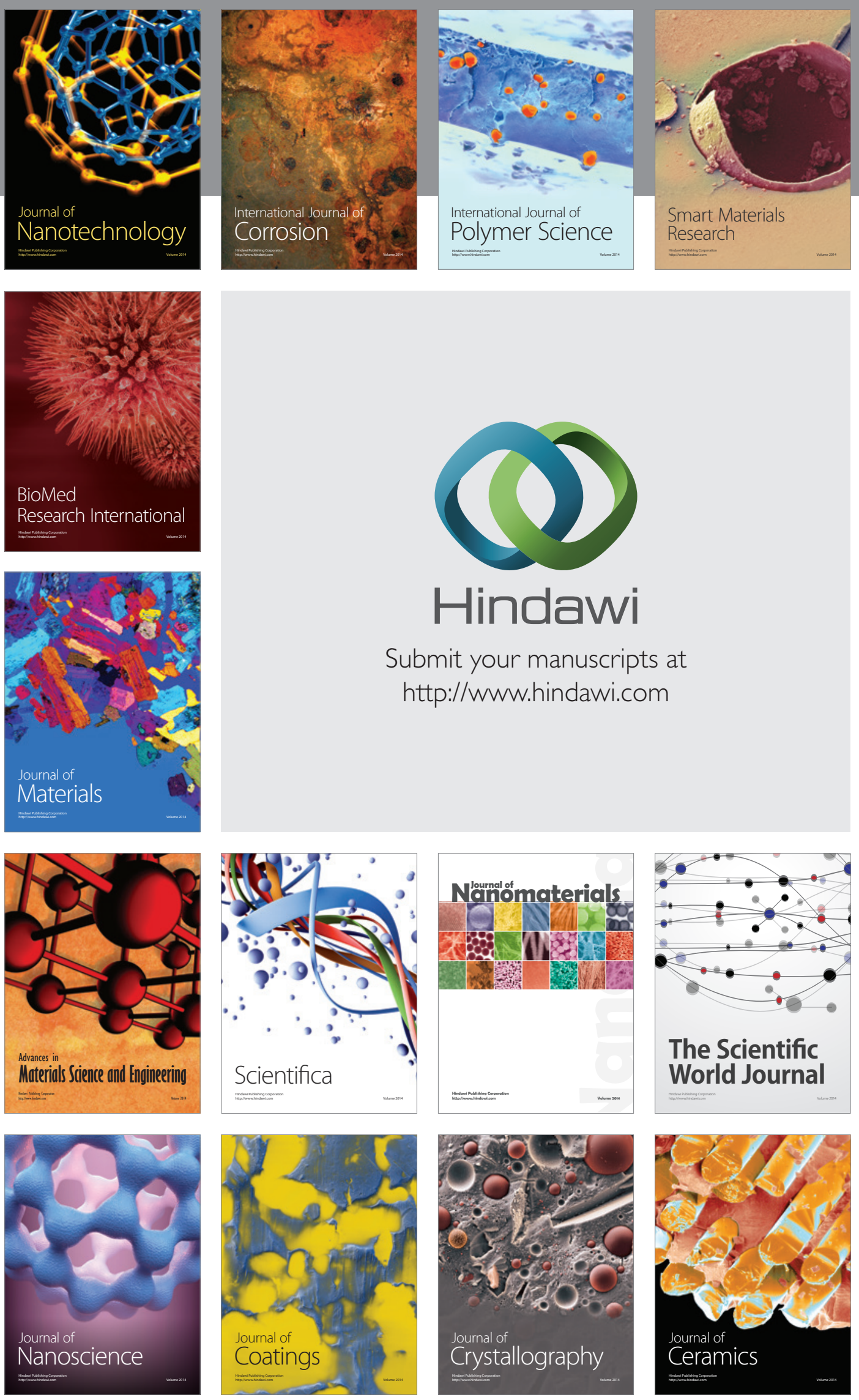

The Scientific World Journal

Submit your manuscripts at

http://www.hindawi.com

\section{World Journal}

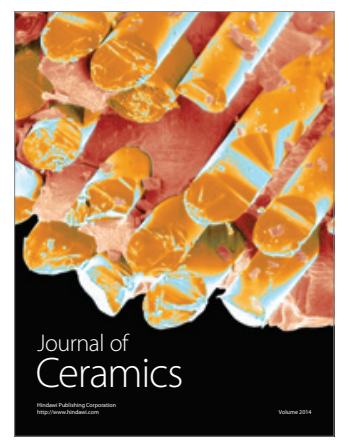

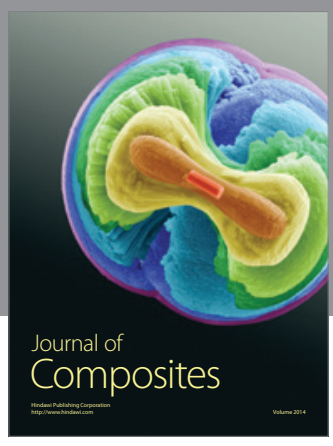
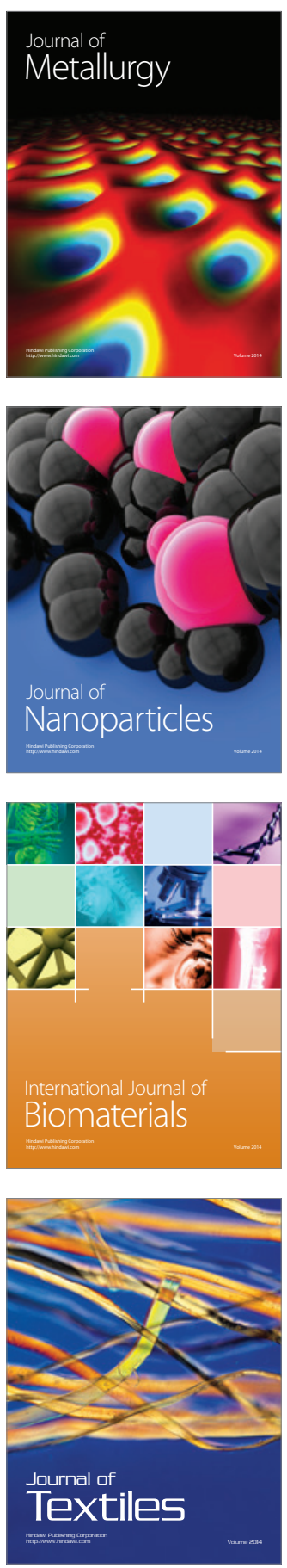